\title{
Investigação sobre as potencialidades da videoaula no que tange às emoções e à formação de memórias
}

\author{
Paulo Marcelo Pedroso Pereira ${ }^{1}$ \\ Carla Marina Costa Paxiúba ${ }^{2}$ \\ Celson Pantoja Lima ${ }^{3}$
}

\section{RESUMO}

Com o advento do Ensino Remoto, as videoaulas tornaram-se parte dos recursos incontornáveis que estão disponíveis para os docentes do mundo todo. Este trabalho apresenta os resultados parciais de uma pesquisa em andamento que busca investigar as emoções durante a visualização de videoaulas preparadas com base na Teoria da Carga Cognitiva (TCC), bem como identificar os elementos armazenados na memória de trabalho. Para isso, os 28 participantes foram distribuídos em 3 grupos. 0 primeiro grupo assistiu a uma videoaula fora das diretrizes da TCC e o segundo dentro das diretrizes. O grupo controle assistiu a uma videoaula pronta. Um software capturou as expressões faciais para converter em emoções. Após as videoaulas, um questionário com perguntas abertas foi respondido. Preliminarmente, verificou-se que não houve diferença estatisticamente significativa em relação às emoções $(p>0,05)$ nos 3 cenários, porém, a média do número de elementos armazenados após a videoaula 2 foi $91 \%$ maior que a videoaula $1(p=0,0054)$.

Palavras-chave: Videoaula. Emoções. Memória de Trabalho.

\footnotetext{
${ }^{1}$ pmpp2004@hotmail.com - Universidade Federal do Oeste do Pará

2 carlamarina@gmail.com - Universidade Federal do Oeste do Pará

${ }^{3}$ celson.ufopa@gmail.com - Universidade Federal do Oeste do Pará
} 


\section{Research on the potentialities of video lesson regarding emoticons and memory formation}

\section{ABSTRACT}

With the advent of Remote Teaching, video lessons have become part of the unavoidable resources that are available to teachers around the world. This work presents the partial results of an ongoing research to investigate emotions during the visualization of video lessons prepared based on the Cognitive Load Theory (CLT), and identify the elements stored in working memory. Twenty-eight participants were divided into three groups. The first watched a video lesson outside the CLT guidelines and the second within the guidelines. The control group watched a video lesson ready. A software captured facial expressions to convert into emotions. After the video lessons, a questionnaire with open questions was answered. Preliminarily, it was found that there was no statistically significant difference in relation to emotions $(p>0.05)$ in the three scenarios, however, the average number of elements stored after video lesson 2 was $91 \%$ higher than video lesson $1(p=0.0054)$.

Keywords: Video lessons. Emotions. Working Memory. 
Com as limitações impostas pela pandemia de Covid-19 em 2020, sistemas educacionais do mundo inteiro se viram em meio ao desafio de continuar as aulas e fazer chegar o conhecimento aos alunos de todos os níveis de ensino. Vários recursos e ferramentas de tecnologias de informação e comunicação foram sendo aprimorados e disseminados no âmbito escolar, com o propósito de auxiliar o trabalho dos professores, sendo que um desses passou a receber atenção relevante por apresentar vantagens como flexibilidade, uso de recursos multimídia e a possibilidade de fornecer aos estudantes a visão geral dos conteúdos curriculares: a videoaula.

Apesar disso, a videoaula precisa ser elaborada com determinadas diretrizes, de forma a potencializar o processamento das informações, considerando a capacidade de percepção audiovisual dos alunos, a interligação entre informação recebida e o que já está armazenado em memórias duradouras, a atenção que é capaz de despertar e, de alguma forma, as emoções que podem ser capazes de estimular aos que estão assistindo ao conteúdo trabalhado.

Esta pesquisa em andamento, aprovada por um Comitê de Ética com o parecer de $n^{\circ}$ 4.731.463, tem como ponto central a inserção de diretrizes da Teoria da Carga Cognitiva (TCC) no âmbito da Aprendizagem Multimídia, mais especificamente na elaboração de videoaulas. A Aprendizagem Multimídia considera o uso de estímulos visuais e auditivos para melhorar a aprendizagem, lançando mão de recursos multimídia (MAYER, 2009).

Tendo como objeto principal a sobrecarga na Memória de Trabalho (MT), a TCC estabelece diretrizes que contribuem para a potencialização desse tipo de memória, que se constitui como um sistema cognitivo que permite ao indivíduo relacionar, em tempo real, as informações disponíveis no ambiente com aquelas armazenadas na memória de longo prazo e tomar decisões otimizadas em função dos próprios objetivos (ZAR; GALERA, 2018). As evidências encontradas mostraram a capacidade de se armazenar $7 \pm 2$ itens - não relacionados entre si simultaneamente na MT. Essa limitação foi verificada por Miller em 1956 (MILLER, 1956; COWAN, 2009). A MT possui uma duração de aproximadamente 18 segundos, sendo que, de acordo com experimentos realizados, após esse período, somente $10 \%$ das informações recebidas puderam ser relembradas (PETERSON; PETERSON, 1959; SOLSO, 1995). Uma vez excedidos os limites da capacidade da MT, o raciocínio e a aprendizagem ficam abaixo do desempenho esperado (SHAH; MIYAKE, 2003).

Os experimentos delineados para o desenvolvimento da TCC buscaram medir a carga cognitiva utilizada pelos sujeitos para a realização de tarefas, considerando os seguintes métodos: autoavaliações, medidas fisiológicas e tarefas secundárias. Por meio da autoavaliação, os próprios sujeitos indicam o esforço mental que estão dispensando para a realização de tarefas. Medidas fisiológicas não são medidas diretas da carga cognitiva, pois apresentam uma ligação causal indireta com a carga, podendo, por exemplo, ser medido o estresse no momento da realização de determinado esforço cognitivo. A análise de tarefas secundárias é baseada na limitação dos recursos cognitivos. Em razão dessa limitação, a execução de uma tarefa afeta o desempenho da outra. Assim, a análise do desempenho de uma tarefa permite inferir o nível de 
demanda cognitiva associado à outra tarefa (SOUZA, 2010). E no caso das emoções, surge a indagação de como se dá a relação entre a carga cognitiva imposta na elaboração de uma videoaula e o despertar de diferentes emoções nos sujeitos, e quais evidências esta relação pode fornecer acerca da quantidade de itens relacionados à referida videoaula a serem armazenados na MT?

As emoções são fenômenos que se manifestam por meio de alterações na fisiologia e nos processos mentais e mobilizam os recursos cognitivos existentes, como atenção e percepção. Nos momentos em que o indivíduo experimenta uma carga emocional, fica mais vigilante e sua atenção se volta para os detalhes considerados importantes, pois as emoções controlam os processos motivacionais (COSENZA; GUERRA, 2011).

Diante do contexto, o recorte aqui apresentado de uma pesquisa atualmente em curso objetiva investigar as emoções prevalentes durante a visualização de videoaulas preparadas a partir de diretrizes da TCC, considerando contextos de sobrecarga e não sobrecarga da MT, bem como seus efeitos no processo de formação desse tipo de memória.

Este artigo está estruturado como se segue: na seção 2, tem-se o referencial teórico com foco nos conceitos de Emoções, Memórias, Teoria da Carga Cognitiva e Aprendizagem Multimídia; a seção 3 apresenta os procedimentos metodológicos para a realização dos experimentos; na seção 4, tem-se a apresentação e discussão dos resultados, concluindo, na seção 5, com as considerações finais, elencando as próximas etapas da pesquisa.

\section{REFERENCIAL TEÓRICO}

A base teórica deste trabalho, descrita nas subseções a seguir, está situada nos pressupostos das neurociências, no que tange aos conceitos de emoções, memórias e aprendizagem, e também na Teoria da Carga Cognitiva, com ênfase na aprendizagem multimídia e nas diretrizes necessárias para que se evite a sobrecarga na memória de trabalho.

\subsection{Conceituando Emoções}

Segundo Kandel et al. (2014), o termo emoção é utilizado comumente de duas formas: na primeira, refere-se a respostas fisiológicas a certos tipos de estímulos; na segunda, refere-se a experiências conscientes, denominadas sentimentos, que frequentemente acompanham essas respostas do organismo. Os autores chamam atenção para a necessidade de se distinguir conscientemente esses dois estados.

Neste trabalho, utiliza-se o termo emoção para se referir ao primeiro desses dois estados: o conjunto de respostas fisiológicas que ocorre mais ou menos inconscientemente quando o encéfalo detecta certas situações desafiadoras. Essas respostas fisiológicas automáticas ocorrem tanto no encéfalo quanto no resto do corpo. No encéfalo, envolvem mudanças nos níveis de alerta e nas funções cognitivas, como atenção, processamento da memória e estratégias de decisão. No restante do corpo, envolvem respostas endócrinas, autônomas e musculoesqueléticas (KANDEL et al., 2014). 


\subsection{Aprendizagem e Memórias}

Sobre a aprendizagem, Kandel et al. (2014) referem como uma mudança no comportamento que resulta da aquisição de conhecimento acerca do mundo, e a memória é o processo pelo qual esse conhecimento é codificado, armazenado e posteriormente evocado. Portanto, para discutir aprendizagem é necessário que se considere o significado e funcionamento das memórias.

Segundo os autores, há diversas formas de aprendizagem e memória, cada qual com suas próprias propriedades cognitivas, mediadas por sistemas encefálicos específicos. Argumentam que a memória pode ser dividida em processos separados: codificação, armazenamento, consolidação e evocação. A codificação refere-se à conexão entre o novo e o preexistente na memória; o armazenamento como sendo o mecanismo de retenção da memória; a consolidação envolve expressão de genes e síntese proteica para alterações estruturais nas sinapses, buscando a estabilidade da informação; e a evocação é o processo de resgate da informação. Por fim, os autores ainda discorrem que imperfeições e erros na evocação dão pistas acerca da natureza e da função da aprendizagem e da memória. Neste trabalho, considera-se o estágio de codificação, por estar vinculado à MT.

\subsection{Teoria da Carga Cognitiva (TCC)}

A TCC parte da ideia, experimentalmente verificada (SWELLER, 1988; CHANDLER; SWELLER, 1991), da existência de uma carga cognitiva necessária para a compreensão das informações durante o processo de aprendizagem. Tais informações são processadas, inicialmente, pela MT, que possui limitações em termos de duração e capacidade de armazenamento. Assim, o ponto central da TCC é considerar a necessidade de não sobrecarga da MT, para que não haja comprometimento na aprendizagem do sujeito (CLARK; NGUYEN; SWELLER, 2006).

A TCC considera a forma como os recursos cognitivos são usados durante o processo de aprendizagem. Muitas dessas formas são desenvolvidas por métodos de instrução que envolvem os sujeitos em atividades cognitivas distantes dos reais objetivos da tarefa em questão. A carga cognitiva gerada por essas atividades, muitas vezes irrelevantes, pode dificultar a aprendizagem (CHANDLER; SWELLER, 1991). Experimentos já evidenciaram a utilização de estratégias que geram consequências negativas para a aprendizagem (COOPER; SWELLER, 1987; WARD; SWELLER, 1990).

\subsection{Memória de Trabalho (MT)}

A MT é uma memória transitória, consciente, onde são armazenadas e processadas as informações necessárias ao desempenho de uma tarefa ou ao entendimento de um conteúdo que esteja sendo aprendido. O funcionamento dessa memória depende de uma coordenação executada, especialmente pela região pré-frontal do córtex cerebral (COSENZA; GUERRA, 2011).

Nos seres humanos, a MT consiste em pelo menos dois subsistemas - um para a informação verbal e outro para a visuoespacial. O funcionamento desses dois subsistemas é coordenado por um terceiro sistema, denominado Processos de Controle Executivo. Acredita-se que os Processos 
de Controle Executivo aloquem recursos de atenção para os subsistemas verbal e visuoespacial, além de monitorar, manipular e atualizar as representações armazenadas (KANDEL et al., 2014).

Utiliza-se o subsistema verbal ao tentar manter informação com base na linguagem falada conscientemente. O subsistema verbal consiste em dois componentes interativos: um armazenado, que representa conhecimento fonológico, e um mecanismo de ensaio - a repetição mental -, que mantém essas representações ativas enquanto se precisa delas. O subsistema visuoespacial retém imagens mentais de objetos visuais e da localização dos objetos no espaço. Acredita-se que o ensaio da informação espacial e relativa a objetos envolva a modulação de tais representações nos córtices parietal, temporal inferior e occipital extraestriatal pelos córtices frontal e pré-motor (KANDEL et al., 2014).

\subsubsection{Evidências Empíricas da Memória de Trabalho}

Em 1885, surge a primeira pesquisa experimental referente ao decaimento da memória com o tempo (SOUZA, 2010). Herman Ebbinghaus sabia que o significado de uma palavra facilita a sua lembrança. Para neutralizar esse efeito facilitador, o pesquisador utilizou sílabas sem sentido. Uma de suas descobertas foi a existência de limites no tempo de armazenamento das informações na MT. A partir daí, foi possível construir um gráfico de decaimento da memória curva do esquecimento (BADDELEY, 1982). O declínio mais rápido dessa curva ocorre nos primeiros 20 minutos. No final dos anos 50 , começou-se a investigar não o esquecimento que ocorre nos primeiros 20 minutos, mas o que ocorre dentro de um intervalo de tempo de alguns segundos.

Peterson e Peterson (1959) desenvolveu uma técnica que consistia na apresentação ao sujeito de um grupo de 3 consoantes, tais como XTM seguido de um número, tal como 582. Pediase ao sujeito que repetisse o número e depois prosseguisse enumerando seus algarismos de trás para frente por algumas vezes, após o que deveria tentar repetir as consoantes. A intenção, ao fazer o sujeito se concentrar nos números em ordem decrescente e em progressão aritmética, pronunciando-os "309, 306, 303", era impedir que pudesse ficar repetindo mentalmente as letras - ABC -, o que impossibilitaria o decaimento natural da lembrança das 3 letras.

Nessas circunstâncias, os participantes esqueciam muito rapidamente. A tarefa secundária, "pedia-se ao sujeito que repetisse o número, digamos 309, e depois prosseguisse, 306, 303 e os seguintes números da sequência decrescente indicada, até a luz vermelha acender" (PETERSON; PETERSON, 1959, p. 194), interferia inibindo a lembrança das 3 letras alguns segundos depois. O pesquisador verificou que após 18 segundos ocorria um esquecimento severo das 3 letras. Esses 18 segundos assim obtidos dessa experiência são frequentemente citados como um indicador da "duração" da MT (SOLSO, 1995).

Em 1956, Miller realizou experimentos baseados também em lembranças de letras e palavras, e concluiu que a MT armazena no máximo $7 \pm 2$ itens simultaneamente. Essa limitação da capacidade significa que não se terá dificuldade em memorizar e repetir uma lista de, por exemplo, 6 itens, mas haverá dificuldade em memorizar uma lista de 10 itens - "novos" e não relacionados entre si -, seja em uma lista de dígitos, letras ou palavras (COWAN, 2009). 


\subsection{Tipos de Carga Cognitiva}

a) Carga Cognitiva Estranha

É a carga cognitiva que em nada contribui para o processo de aprendizagem, causando efeito reverso, ou seja, consumindo parte da capacidade da MT que poderia estar sendo utilizada para a aprendizagem (SOUZA, 2010). A carga cognitiva gerada tanto pelo efeito da atenção dividida quanto pelo efeito redundância é irrelevante para a aprendizagem, é estranha à aprendizagem, por isso é classificada como uma "Carga Cognitiva Estranha" (AYRES; SWELLER, 2005).

b) Carga Cognitiva Intrínseca

A Carga Cognitiva Intrínseca é aquela que diz respeito à natureza/dificuldade intrínseca do conteúdo, que está relacionada ao grau de interatividade entre os elementos que compõem a informação a ser apreendida. Quanto maior for essa interatividade, mais complexo é o conteúdo e maior será a carga cognitiva intrínseca envolvida na aprendizagem (SOUZA, 2010; CLARK; NGUYEN; SWELLER, 2006).

c) Carga Cognitiva Relevante

A Carga Cognitiva Relevante está associada a processos que são relevantes para a aprendizagem, tais como aquisição de esquemas e automatização. É a carga que contribui diretamente para a aprendizagem, por meio da construção das estruturas cognitivas e dos processos que melhoram o desempenho dos aprendizes (VAN MERRIENBOER; KESTER; PAAS, 2006).

\subsection{Efeitos que envolvem a Memória de Trabalho}

a) Efeito da Atenção Dividida

De um modo geral, os materiais didáticos apresentam duas fontes de informação: texto e figuras. Na maioria dos casos, texto e figuras ficam espacialmente separados, obrigando o aprendiz a olhar ora para o texto, ora para a figura, dividindo a atenção. Isso cria uma carga cognitiva, uma vez que, para a compreensão do texto, o aprendiz deve lê-lo, guardá-lo na MT, e então procurar na figura o referente correspondente (SOUZA, 2010; AYRES; SWELLER, 2005). Esse esforço cognitivo é denominado efeito da atenção dividida, verificado experimentalmente por Tarmizini e Sweller (1988).

Os autores discorrem que essa carga cognitiva causada pela separação espacial das duas fontes de informação pode ser eliminada caso seja feita a integração espacial das fontes. Por ser uma carga considerada irrelevante para a aprendizagem, é classificada como uma "Carga Cognitiva Estranha”. A recomendação de fazer a integração espacial das fontes é denominada de Princípio da Atenção Dividida.

b) Efeito Redundância

Após a descoberta do efeito da atenção dividida, verificou-se que não era válido para qualquer tipo de material didático (SOUZA, 2010). O efeito redundância é o surgimento da carga 
cognitiva estranha causada pela apresentação de um conteúdo por meio de texto e figuras, sendo que um repassa a mesma ideia do outro, ou seja, causam redundância na leitura das informações. Experimentalmente, tal efeito foi verificado por Chandler e Sweller (1991).

Eliminando-se a informação redundante, liberam-se esses recursos para a aprendizagem (CHANDLER; SWELLER, 1991). Essa recomendação é denominada de Princípio da Redundância, que afirma que os aprendizes aprendem mais profundamente a partir da animação e narração do que a partir da animação, narração e texto, por exemplo (MORENO, 2004).

c) Efeito Modalidade

A MT possui um sistema de armazenamento para informação auditiva e outro para informação visual (SOUZA, 2010). A capacidade da MT de um aprendiz é efetivamente aumentada quando a aprendizagem se dá por meio de material didático que utiliza a modalidade dual auditiva e visual -, ou seja, dois canais de percepção (MOUSAVI; LOW; SWELLER, 1995). Há evidências de que a eficiência cognitiva aumenta quando se substitui o texto escrito que acompanha uma figura, pelo texto falado (SOUZA, 2010). A eficiência cognitiva conseguida em razão dessa substituição é denominada Efeito Modalidade (CLARK; NGUYEN; SWELLER, 2006). A recomendação para esta adequação na apresentação dos conteúdos é chamada Princípio da Modalidade (SOUZA, 2010).

É mister considerar que se as explicações via áudio apenas reforçam as informações que a própria figura já contém, a duplicação da informação via modo dual é redundante e pode prejudicar a aprendizagem (LEAHY; CHANDLER; SWELLER, 2003). Quando uma informação visual como uma figura precisar de uma explicação, é recomendável que seja via áudio - Princípio da Modalidade - ou texto integrado, para evitar o Efeito da Atenção Dividida (CLARK; NGUYEN; SWELLER, 2006).

\subsection{Diretrizes da Teoria da Carga Cognitiva}

Das 29 diretrizes propostas pela TCC, neste trabalho, foram consideradas 7, as quais estão relacionadas com materiais multimídia - videoaula -, descritas a seguir:

a) Usar diagramas para ajudar na construção de uma compreensão mais profunda Há sólidas pesquisas e razões psicológicas para que os diagramas sejam utilizados no ensino (CLARK; NGUYEN; SWELLER, 2006). Todos os elementos em um visual podem ser vistos simultaneamente, diferente de sentenças que devem ser processadas em sequência. Isso leva a uma menor busca visual em tarefas que envolvem a coordenação de múltiplos elementos espaciais, o que resulta em uma maior eficiência no processamento psicológico. Do mesmo modo, diagramas fornecem uma representação mais explícita das tarefas espaciais. Um diagrama requer menos inferências porque mostra relações espaciais que deveriam ser inferidas a partir de texto (LARKIN; SIMON, 1987). Os autores argumentam que existe uma correspondência mais próxima entre o diagrama e os requisitos da tarefa (CLARK; NGUYEN; SWELLER, 2006).

b) Explicar diagramas com palavras apresentadas em áudio-narração 
A TCC considera que a MT possui múltiplos sistemas de armazenamento: um para informações visuais e outro para auditivas. A utilização do texto falado em uma apresentação canaliza as informações auditivas para o subcomponente auditivo da MT, liberand o o canal visual - subcomponente visual da MT - para o processamento de outras informações, impedindo que haja sobrecarga em ambos os subcomponentes, promovendo um aumento efetivo da capacidade da MT (CLARK; NGUYEN; SWELLER, 2006).

Assim, na apresentação de diagramas ou figuras acompanhadas de explicações em textos, deve-se apresentar o texto como narração - áudio. A eficiência cognitiva conseguida em razão da substituição do texto escrito pelo texto falado é, conforme já visto neste texto, chamada de Efeito Modalidade.

c) Usar sinais para focar a atenção em conteúdos visuais e textuais importantes

Esta diretriz foi verificada experimentalmente por Jeung, Chandler e Sweller (1997). As evidências sugeriram que o efeito modalidade não era alcançado quando os diagramas apresentavam uma alta complexidade. Contudo, pela adição de sinais indicativos nos diagramas, como setas, o efeito modalidade era recuperado.

Nesses experimentos, segundo os autores, foram comparados três formatos diferentes de apresentação de exemplos geométricos: (i) diagramas sem sinais indicativos, explicados por meio de narração falada; (ii) diagramas com sinais indicativos que piscavam, explicados por meio de narração falada; e (iii) diagramas sem pistas, explicados por texto. Para os diagramas complexos, a aprendizagem por meio da narração foi melhor do que a aprendizagem por meio de textos, somente quando a parte relevante do diagrama era indicada por sinais indicativos para chamar a atenção da parte que estava sendo explicada.

d) Integrar o texto explicativo próximo aos visuais correspondentes nas páginas e telas

Esta diretriz está relacionada ao Efeito da Atenção Dividida, conforme já apresentado. Esse efeito foi verificado também, experimentalmente, por Tindall-Ford (TINDALL-FORD; CHANDLER; SWELLER, 1997), quando o pesquisador comparou dois tipos diferentes de apresentações: uma com integração espacial e outra sem. Nesse mesmo experimento, o pesquisador acrescentou mais um formato de apresentação no qual substituiu o texto pelo áudio.

As evidências sugeriram que o texto sem integração possibilitou uma menor aprendizagem. A pesquisa mostrou ainda que, tanto na apresentação com formato integrado quanto na apresentação em que ocorreu a substituição do texto por áudio, os alunos aprenderam duas vezes mais do que na apresentação sem integração. Nesse mesmo estudo, quando se compararam os efeitos sobre a aprendizagem decorrentes da versão em que o áudio foi substituído por texto, com os efeitos da aprendizagem decorrentes da versão na qual havia integração espacial entre texto e partes da figura a ele correspondentes, não houve diferença significativa.

e) Reduzir o conteúdo ao essencial 
Para apresentação inicial do conteúdo, deve-se considerar a limitação da MT para a seleção das informações a serem inseridas no material instrucional, com vistas a não sobrecarga da MT (CLARK; NGUYEN; SWELLER, 2006). Souza (2010) relata o experimento desenvolvido por Mayer, em 1995. Foram comparados os efeitos sobre a aprendizagem de 3 versões de resumos, sobre processos climáticos, que continham um número de palavras diferentes: (i) ilustrações com textos de 50 palavras; (ii) com textos de 100 palavras; e (iii) com textos de 550 palavras. Os resultados sugeriram que o texto contendo o menor número de palavras foi o que produziu maior aprendizagem.

f) Eliminar visuais, texto e áudio estranhos ao conteúdo a ser aprendido

Carga cognitiva estranha ao conteúdo sobrecarrega a MT, conforme já descrito neste projeto. Harp e Mayer (1997) mostraram que se deve eliminar visuais ou áudio que foram incluídos apenas para motivar o aluno, pois, segundo eles, as evidências mostram que o melhor modo de ajudar alunos a gostarem de um conteúdo é contribuir com sua compreensão.

g) Eliminar a redundância nos modos de apresentação do conteúdo

Esta diretriz está relacionada ao Efeito Redundância, conforme já apresentado.

\subsection{Aprendizagem Multimídia}

De acordo com Mayer (2009), os recursos multimídia são alternativas interessantes para melhorar a aprendizagem, tendo em vista que podem fazer uso de estímulos visuais e auditivos. O termo multimídia, segundo Braga et al. (2019, p. 2), pode ser compreendido como um "conjunto de diversos meios técnicos, com o objetivo de apresentar as informações de diversas formas e por meio de diferentes modalidades sensoriais". Desse modo, o recurso multimídia pode ser percebido a partir de três níveis: nível técnico - vinculado às ferramentas que são condutoras de sinais, como computadores e monitores; nível semiótico - relacionado à forma de apresentação desses sinais, como textos, imagens e sons e; nível sensorial - vinculado à modalidade de recebimento de sinais, visual ou auditiva. No âmbito desta pesquisa, a exibição de videoaulas por meio de computadores, que são os condutores de sinais, configura-se como nível técnico. Já a forma como a videoaula apresenta as ideias ou informações - seja com narração, textos ou figuras - corresponde ao nível semiótico da aula. Estas informações apresentadas textualmente, narração ou texto escrito; ou visualmente, figuras ou diagramas, serão recebidas pelos estudantes através dos canais visual ou auditivo, representam o nível sensorial dos recursos multimídia.

Schnotz e Lowe (2003) destacam que os três níveis apresentados são igualmente relevantes para o processo de aprendizagem, uma vez que impactam diretamente o processamento da informação. Os autores argumentam que diversas ferramentas multimídia voltadas ao ensino, apesar de possuírem a possibilidade de utilização de ambos os canais semióticos e sensoriais visual ou auditivo -, utilizam majoritariamente um dos canais, por exemplo, apresentando textos escritos e imagens simultaneamente, o que pode gerar sobrecarga na MT.

Mayer (2005) discorre que a aprendizagem multimídia é baseada em três pressupostos: o pressuposto do canal duplo, no qual o sujeito possui canais de processamento de informação 
separados - visual e verbal -; o da capacidade limitada - relacionado à MT -, no qual há limitação no processamento de informação em cada um dos canais; e o pressuposto da aprendizagem ativa, no qual há processamento cognitivo essencial em ambos os canais.

Segundo Mayer (2005), a aprendizagem multimídia se dá por meio de animação e narração, processada em três memórias: sensorial, de trabalho e de longo tempo. As informações são captadas pela memória sensorial por meio dos olhos - palavras e imagens - e ouvidos - palavras -; em seguida, são processadas e selecionadas no canal auditivo, onde ocorre a seleção de palavras e imagens. Na MT, há uma organização entre imagens e palavras formando os modelos pictorial e verbal. Por fim, ocorre a integração das informações que, juntamente com o conhecimento prévio, constrói-se a memória de longo prazo. Argumenta ainda que as informações armazenadas na memória de longo prazo afetam as percepções do mundo dos sujeitos e influenciam nas tomadas de decisão.

Os princípios da Aprendizagem Multimídia foram elaborados a partir dos pressupostos da TCC, e consideram as formas de elaborar os recursos multimídia para que os alunos aprendam melhor. Para o princípio multimídia, isso ocorre quando se combinam palavras e imagens do que apenas palavras; para o princípio da contiguidade temporal, isso ocorre quando palavras e imagens são apresentadas simultaneamente em vez de sucessivamente; para o princípio da coerência, ocorre quando palavras, imagens ou sons não relevantes ao assunto são excluídos; para o princípio da personalização, quando as palavras são no estilo de conversação em vez de estilo formal; já para o princípio da voz, isso ocorre quando a narração em aulas multimídia é falada em voz humana em vez de voz de máquina; para o princípio da imagem, não de forma necessária a imagem do professor deve ser colocada no material. Os princípios da redundância, da modalidade, da atenção dividida e da sinalização já foram enunciados neste trabalho.

\section{3}

\section{METODOLOGIA DA PESQUISA}

O desenho metodológico está situado nos pressupostos da Pesquisa Experimental. O delineamento estabelecido tem como base Lazar, Feng e Hochheiser (2017). Neste recorte, o objeto de estudo foram as emoções prevalentes no momento da visualização de videoaulas elaboradas fora das diretrizes da TCC - videoaula 1 - e dentro das diretrizes - videoaula 2 -, bem como a formação da MT alcançada após a visualização dos vídeos.

Fizeram parte desta fase dos experimentos 28 estudantes de cursos de graduação de uma faculdade privada localizada no Oeste do Pará, Brasil. Os sujeitos foram divididos de forma randômica em 3 grupos, sendo dois experimentais e um controle. Os 3 grupos assistiram à videoaula do mesmo conteúdo - Estudos Epidemiológicos -, com duração de 11 minutos.

A videoaula 3 (INTRODUÇÃO, 2014) foi convencionada para o grupo controle, e serviu de base para a construção das demais videoaulas, a fim de manter o mesmo conteúdo. A videoaula 1 (ESTUDOS, 2021a) foi construída de forma a sobrecarregar a MT, com a presença de elementos que geram Efeito redundância, Efeito modalidade, carga cognitiva irrelevante e Efeito da atenção dividida. Na videoaula 2 (ESTUDOS, 2021b), foram inseridas diretrizes da TCC como o uso de diagramas, uso de sinais e redução do conteúdo ao essencial, conforme exemplificadas nas figuras de 1 a 4. 
Figura 1: Slide com marcações sobre os efeitos que sobrecarregam a MT (videoaula 1)

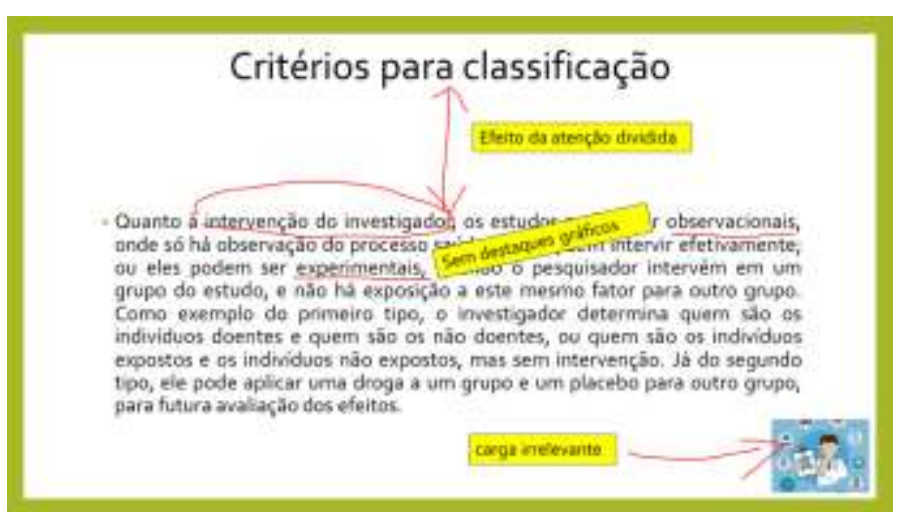

Fonte: Dados da pesquisa (2021).

Figura 2: Slide com marcações das diretrizes da TCC (videoaula 2)

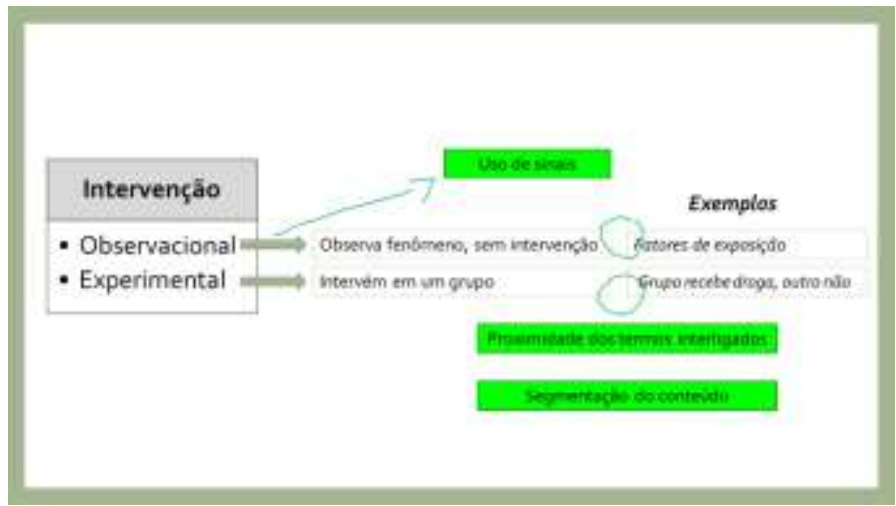

Fonte: Dados da pesquisa (2021).

Figura 3: Slide com marcações sobre os efeitos que sobrecarregam a MT (videoaula 1)

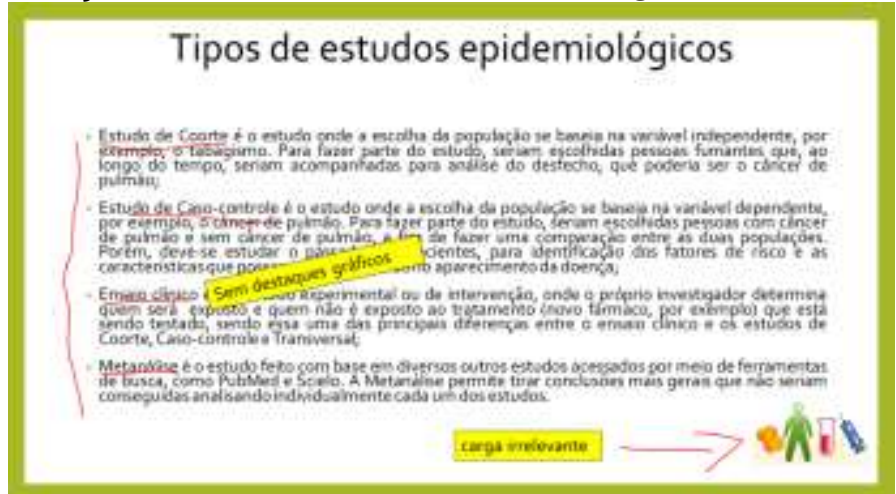

Fonte: Dados da pesquisa (2021). 
Figura 4: Slide com marcações das diretrizes da TCC (videoaula 2)

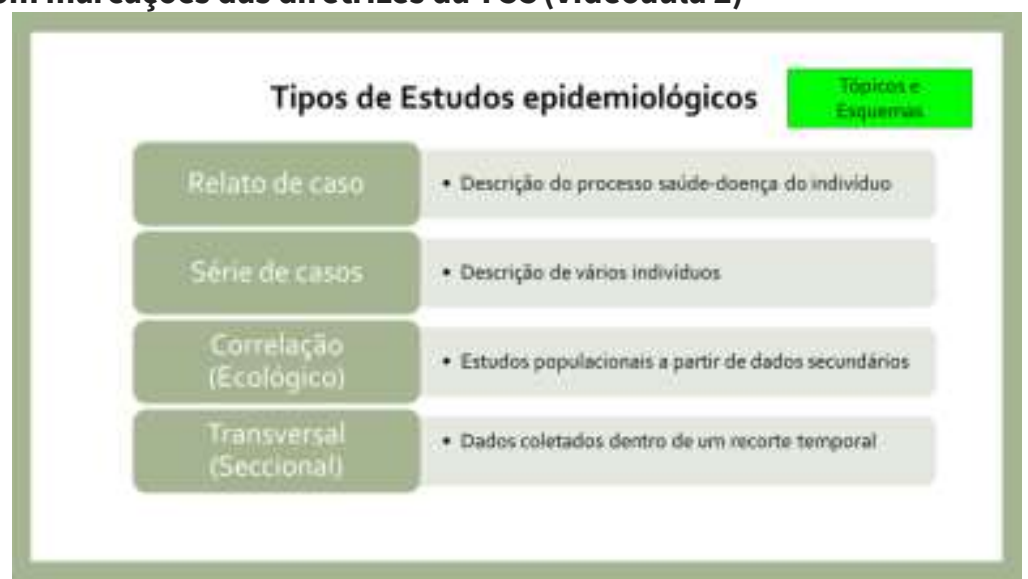

Fonte: Dados da pesquisa (2021).

Destaca-se que o áudio inserido na videoaula 1 narrava a repetição do que estava escrito no slide e o áudio inserido na videoaula 2 narrava a explicação do que estava sintetizado no slide. Imediatamente após a visualização das videoaulas, os estudantes responderam a um questionário com seis perguntas abertas -respostas curtas -, a fim de verificar quais elementos do conteúdo ficariam armazenados na MT.

Para a identificação das emoções, foi utilizado o software Cara de Aprende (CADAP), desenvolvido para capturar as expressões faciais dos estudantes durante a visualização de videoaulas. O mecanismo utilizado para o reconhecimento das expressões faciais é composto por um software que é responsável por reconhecer as expressões faciais, de forma a traduzir em uma das sete emoções: alegria, medo, raiva, desgosto, tristeza, desprezo e surpresa (PAXIÚBA; LIMA, 2019). As emoções variam em uma escala de 0 a 100, sendo 0 indicador de emoção ausente e 100 emoção no ápice.

Como Metodologia de Análise de dados, após verificação da normalidade - Teste ShapiroWilk, usado para amostras com $2<n<51$ - com o uso do software estatístico BioEstat versão 5.3, foi utilizado o Teste não-paramétrico de Kruskal-Wallis para a comparação entre os escores dos 3 grupos, em virtude de os dados terem sido verificados como não normais. 0 teste destina-se a comparar 3 ou mais amostras independentes do mesmo tamanho ou desiguais, cujos escores devem ser mensurados, pelo menos, a nível ordinal (LEVIN, 1987).

\section{RESULTADOS E DISCUSSÕES}

Após aplicação dos testes estatísticos devidos, foram encontrados os seguintes resultados, em que as tabelas apresentam os escores médios de cada uma das emoções decodificadas pelo CADAP, divididas em colunas que representam os cenários do experimento, sendo que foi utilizado o termo Aula 1 para designar a videoaula 1, e assim sucessivamente; e os quadros trazem os resultados dos testes com seus respectivos valores de p, a um nível de significância de 5\%. 
Tabela 1: Escores médios/Medo

\begin{tabular}{ccc}
\hline Aula 1 & Aula 2 & Aula 3 \\
\hline 0.07 & 0.12 & 0.12 \\
0.00 & 0.00 & 0.13 \\
0.00 & 0.00 & 0.02 \\
0.22 & 0.12 & 0.00 \\
0.01 & 0.00 & 0.00 \\
0.00 & 5.29 & 0.00 \\
0.11 & 0.00 & 0.00 \\
0.01 & 0.00 & 0.13 \\
0.04 & 0.00 & \\
\hline
\end{tabular}

Fonte: Dados da pesquisa (2021).

Quadro 1 :Teste Kruskal-Wallis/Medo

\begin{tabular}{|r|l|}
\hline \multicolumn{1}{|c|}{ Variáveis } & Resultados \\
\hline$H=$ & 0,3930 \\
\hline Graus de liberdade $=$ & 2 \\
\hline (p) Kruskal- Wallis $=$ & 0,8216 \\
\hline
\end{tabular}

Fonte: BioEstat versão 5.3

Tabela 3: Escores médios/Raiva

\begin{tabular}{lll}
\hline Aula 1 & Aula 2 & Aula 3 \\
\hline 0.08 & 0.04 & 0.03 \\
0.00 & 0.24 & 0.01 \\
0.13 & 0.01 & 2.23 \\
0.01 & 0.15 & 0.00 \\
0.20 & 0.00 & 0.01 \\
0.02 & 0.15 & 0.13 \\
0.01 & 0.14 & 0.07 \\
0.10 & 0.00 & 0.01 \\
0.13 & 0.05 & \\
\hline
\end{tabular}

Fonte: Dados da pesquisa (2021).

Quadro 3: Teste Kruskal-Wallis/Raiva

\begin{tabular}{|r|l|}
\hline Variáveis & Resultados \\
\hline $\mathrm{H}=$ & 0,4258 \\
\hline Graus de liberdade $=$ & 2 \\
\hline (p) Kruskal-Wallis $=$ & 0,8082 \\
\hline
\end{tabular}

Fonte: BioEstat versão 5.3
Tabela 2: Escores médios/Alegria

\begin{tabular}{lll}
\hline Aula 1 & Aula 2 & Aula 3 \\
\hline 1.54 & 0.39 & 1.89 \\
0.15 & 0.10 & 0.22 \\
0.0 & 0.09 & 0.46 \\
1.18 & 0.68 & 0.00 \\
0.48 & 0.00 & 0.10 \\
0.59 & 0.24 & 0.03 \\
0.0 & 0.31 & 0.13 \\
0.79 & 0.08 & 0.15 \\
1.19 & 0.00 & \\
\hline
\end{tabular}

Fonte: Dados da pesquisa (2021).

Quadro 2 :Teste Kruskal-Wallis/Alegria

\begin{tabular}{|r|l|}
\hline \multicolumn{1}{|c|}{ Variáveis } & Resultados \\
\hline $\mathrm{H}=$ & 2,7673 \\
\hline Graus de liberdade $=$ & 2 \\
\hline (p) Kruskal-Wallis $=$ & 0,2507 \\
\hline
\end{tabular}

Fonte: BioEstat versão 5.3

Tabela 4: Escores médios/Tristeza

\begin{tabular}{lll}
\hline Aula 1 & Aula 2 & Aula 3 \\
\hline 0.01 & 0.07 & 1.15 \\
0.06 & 0.00 & 0.00 \\
0.06 & 0.00 & 1.27 \\
0.00 & 0.04 & 0.11 \\
0.01 & 0.26 & 0.00 \\
0.10 & 0.00 & 0.00 \\
0.40 & 0.28 & 0.26 \\
0.16 & 0.40 & 0.00 \\
0.28 & 0.00 & \\
\hline
\end{tabular}

Fonte: Dados da pesquisa (2021).

Quadro 4: Teste Kruskal-Wallis/Tristeza

\begin{tabular}{|r|l|}
\hline Variáveis & Resultados \\
\hline $\mathrm{H}=$ & 0,4006 \\
\hline Graus de liberdade $=$ & 2 \\
\hline (p) Kruskal-Wallis $=$ & 0,8185 \\
\hline
\end{tabular}

Fonte: BioEstat versão 5.3 
Tabela 5: Escores médios/Desgosto

\begin{tabular}{lll}
\hline Aula 1 & Aula 2 & Aula 3 \\
\hline 0.53 & 0.04 & 0.41 \\
0.31 & 0.06 & 0.14 \\
0.58 & 0.16 & 2.23 \\
0.51 & 0.31 & 0.81 \\
0.06 & 0.04 & 0.54 \\
0.08 & 0.01 & 0.01 \\
2.59 & 0.77 & 0.11 \\
0.55 & 0.29 & 0.02 \\
0.06 & 0.02 & \\
\hline
\end{tabular}

Fonte: Dados da pesquisa (2021).

Quadro 5: Teste Kruskal-Wallis/Desgosto

\begin{tabular}{|r|l|}
\hline \multicolumn{1}{|c|}{ Variáveis } & $\underline{\text { Resultados }}$ \\
\hline$\underline{H}=$ & $\underline{3,3149}$ \\
\hline$\underline{\text { Graus de liberdade }=}$ & $\underline{2}$ \\
\hline (p) Kruskal-Wallis $=$ & $\underline{0,1906}$ \\
\hline
\end{tabular}

Fonte: BioEstat versão 5.3 (2021).
Tabela 6: Escores médios/Desprezo

\begin{tabular}{lll}
\hline Aula 1 & Aula 2 & Aula 3 \\
\hline 0.86 & 0.05 & 1.14 \\
4.36 & 2.08 & 11.58 \\
1.01 & 2.66 & 6.27 \\
0.31 & 1.05 & 0.03 \\
0.24 & 0.00 & 0.22 \\
0.48 & 0.00 & 0.06 \\
7.26 & 0.04 & 0.09 \\
0.34 & 0.00 & 1.64 \\
0.20 & 0.01 &
\end{tabular}

Fonte: Dados da pesquisa (2021).

Quadro 6: Teste Kruskal-Wallis/Desprezo

\begin{tabular}{|r|l|}
\hline \multicolumn{1}{|c|}{ Variáveis } & $\underline{\text { Resultados }}$ \\
\hline$\underline{\mathrm{H}=}$ & $\underline{4,1387}$ \\
\hline$\underline{\text { Graus de liberdade }=}$ & $\underline{2}$ \\
\hline (p) Kruskal-Wallis $=$ & $\underline{0,1263}$ \\
\hline
\end{tabular}

Fonte: BioEstat versão 5.3 (2021).

Tabela 7: Escores médios/Surpresa

\begin{tabular}{lll}
\hline Aula 1 & Aula 2 & Aula 3 \\
\hline 1.52 & 0.5 & 4.74 \\
2.22 & 0.15 & 7.64 \\
1.05 & 1.86 & 4.54 \\
0.73 & 0.47 & 1.25 \\
0.7 & 0.21 & 0.15 \\
1.34 & 0.26 & 0.08 \\
3.86 & 1.01 & 0.59 \\
0.49 & 0.77 & 1.45 \\
0.65 & 0.03 & \\
\hline
\end{tabular}

Fonte: Dados da pesquisa (2021).

Quadro 7: Teste Kruskal-Wallis/Surpresa

\begin{tabular}{|c|c|}
\hline Variáveis & Resultados \\
\hline $\mathrm{H}=$ & 4,6498 \\
\hline Graus de liberdade $=$ & 2 \\
\hline (p) Kruskal-Wallis $=$ & 0,0978 \\
\hline
\end{tabular}

Fonte: BioEstat versão 5.3 (2021).

Conforme verificado nas Tabelas de 1 a 7 e nos quadros de 1 a 7 , os dados apontam que não houve diferença estatisticamente significativa $(p>0,05)$ em relação aos escores médios das 
emoções - medo, alegria, raiva, tristeza, desgosto, desprezo e surpresa - decodificadas pelo software quando comparados os três cenários do experimento.

A Tabela 8 apresenta o número de itens corretos do questionário respondido pelos participantes após assistirem às videoaulas nos 3 cenários do experimento. Tal questionário foi composto por 6 perguntas abertas, mas que requeriam respostas curtas e diretas, totalizando 9 itens para serem lembrados.

Tabela 8: $\mathrm{N}^{\circ}$ de acertos às perguntas do questionário

\begin{tabular}{ccc}
\hline Aula 1 & Aula 2 & Aula 3 \\
\hline 2 & 4 & 1 \\
1 & 4 & 4 \\
3 & 4 & 5 \\
1 & 5 & 8 \\
2 & 8 & 3 \\
3 & 2 & 4 \\
2 & 4 & 8 \\
4 & 4 & 3 \\
2 & 3 & 6 \\
2 & & \\
\hline
\end{tabular}

Fonte: Dados da pesquisa (2021).

A Tabela 9 apresenta os resultados do teste de normalidade dos dados:

Tabela 9: Aplicação do Teste de Normalidade Shapiro-Wilk

\begin{tabular}{r|c|c|c}
\hline Variáveis & Aula 1 & Aula 2 & Aula 3 \\
\hline Tam. da Amostra $=$ & 10 & 9 & 9 \\
Média $=$ & 2,2000 & 4,2222 & 4,6667 \\
Desvio padrão $=$ & 0,9189 & 1,6415 & 2,3452 \\
W $=$ & 0,8854 & 0,8112 & 0,9400 \\
$\mathrm{p}=$ & 0,1949 & 0,0359 & 0,5538 \\
\hline
\end{tabular}

Fonte: BioEstat versão 5.3 (2021).

Como uma das amostras apresentou comportamento não normal dos dados ( $p=0,0359)$, seguiu-se para aplicação do Teste de Kruskal-Wallis, considerando as seguintes hipóteses a serem testadas:

a) $\mathrm{H}_{0}$ : Não há diferença estatisticamente significativa entre os grupos em relação ao número de acertos do questionário;

b) $\mathrm{H}_{1}$ : Há diferença estatisticamente significativa entre os grupos em relação ao número de acertos do questionário.

A Tabela 10 apresenta os resultados, após a aplicação do teste de Kruskal-Wallis: 
Tabela 10: Teste Kruskal-Wallis/Itens armazenados na MT

\begin{tabular}{|c|c|c|c|c|}
\hline Variáveis & \multicolumn{4}{|c|}{ Resultados } \\
\hline $\mathrm{H}=$ & 10,4502 & & & \\
\hline Graus de liberdade $=$ & 2 & & & \\
\hline $\mathrm{p}($ Kruskal-Wallis $)=$ & 0,0054 & & & \\
\hline $\mathrm{R} 1=$ & 79,0000 & & & \\
\hline $\mathrm{R} 2=$ & 161,5000 & & & \\
\hline $\mathrm{R} 3=$ & 165,5000 & & & \\
\hline R1 (posto médio) = & 7,9000 & & & \\
\hline R2 (posto médio) = & 17,9444 & & & \\
\hline R3 (posto médio) = & 18,3889 & & & \\
\hline $\begin{array}{c}\text { Comparações } \\
\text { (Método de Dunn) }\end{array}$ & Dif. Postos & z calculado & z crítico & $p$ \\
\hline Postos médios 1 e 2 & 10,0444 & 2,6576 & 2,394 & $<0,05$ \\
\hline Postos médios 1 e 3 & 10,4889 & 2,7752 & 2,394 & $<0,05$ \\
\hline Postos médios 2 e 3 & 0,4444 & 0,1146 & 2,394 & ns \\
\hline
\end{tabular}

Fonte: BioEstat versão 5.3 (2021).

Os dados apontam que, para um $\mathrm{p}=0,0054$, deve-se rejeitar $\mathrm{H}_{0}$ e aceitar $\mathrm{H}_{1}$, ou seja, houve diferença estatisticamente significativa no número de itens corretos respondidos pelos participantes da pesquisa, quando comparados os 3 cenários do experimento. Para a comparação dos grupos 2 a 2, foi aplicado o Método de Dunn, que é utilizado após o teste de Kruskal-Wallis (K-W), se e somente se o teste de K-W permite rejeitar $\mathrm{H}_{0}$ (LEVIN, 1987). A partir dos testes realizados, verificou-se que houve diferença estatisticamente significativa entre os grupos 1 e 2 ( $p<0,05)$ e entre os grupos 1 e $3(p<0,05)$, considerando nível de significância de $5 \%$. Já entre os grupos 2 e 3 , verifica-se que não houve diferença estatisticamente significativa.

A média de acertos do grupo 1 , que assistiu à videoaula que apresentava elementos que sobrecarregavam a MT, considerando as diretrizes da TCC, foi de 2,2, ao passo que a média do grupo 2, que assistiu à videoaula construída dentro das diretrizes da teoria, foi de 4,2, ou seja, houve aumento de cerca de $91 \%$ na média de itens lembrados corretamente imediatamente após a visualização das videoaulas, corroborando com pesquisas que mostram as evidências de melhorias no desempenho da MT quando esta não sofre sobrecarga cognitiva (CLARK; NGUYEN; SWELLER, 2006).

Alguns apontamentos, indicativos de tendência ou possibilidades de reflexão, podem ser feitos a partir dos resultados ora apresentados: os dados sugerem que, independentemente da configuração das videoaulas que foram apresentadas, seja fora das diretrizes, dentro das diretrizes ou ainda uma videoaula usual pronta retirada do YouTube, a média dos escores relativos às emoções decodificadas pelo software não apresentou diferença significativa. É possível também discutir a influência direta do formato da videoaula, com seus recursos visual e auditivo, no nível de retenção de conteúdo dos participantes, ainda que tenham sido evocadas, em momentos distintos, emoções com valência negativa ou positiva. Constatou-se também que, mesmo as videoaulas gerando um certo nível de evocação de emoções básicas, não foi possível verificar um grau de interferência destas na retenção de itens na MT, uma vez que os resultados não apontaram diferença significativa nos cenários do experimento. Como possibilidade de reflexão, os dados podem sugerir também que as videoaulas dentro das diretrizes da TCC, ou seja, 
que não sobrecarregam a MT, devem possuir uma tolerância para evocação de emoções negativas de tal forma que, quando comparado ao cenário onde há sobrecarga cognitiva, o nível de retenção na MT é potencializado.

\section{CONSIDERAÇÕES FINAIS}

No atual momento de isolamento social, em que a participação do professor no compartilhamento de conhecimento foi transportada quase que exclusivamente para as atividades remotas, o uso das tecnologias de informação e comunicação se tornou imperativo no complexo processo de ensino e aprendizagem, gerando ainda mais protagonismo na qualidade do material didático que é utilizado para apoiar este processo.

Dentre as possibilidades de material de estudo, as videoaulas se mostram com potencial para garantir que os alunos tenham acesso às explanações dos professores, aliando recursos audiovisuais que podem ajudar no entendimento dos conteúdos curriculares que estão sendo compartilhados. No entanto, é necessário compreender que todos os elementos presentes em uma videoaula, seja textual - escrito ou narrado - ou com uso de imagens - gráficos, esquemas, diagramas ou figuras - mobilizam recursos cognitivos e emoções que podem contribuir em maior ou menor escala para a aprendizagem.

Até o momento, esta pesquisa conseguiu evidenciar a presença de emoções de valência positiva e negativa durante a visualização de videoaulas elaboradas dentro e fora de diretrizes teóricas que podem potencializar a MT, sendo esta a responsável pelo processamento cognitivo inicial das informações que chegam aos estudantes. Os experimentos realizados evidenciaram não haver diferença significativa nos escores médios de emoções nos 3 cenários do experimento. Porém, os dados mostraram uma média de acertos às perguntas do questionário cerca de $90 \%$ maior, comparando a videoaula 2 em relação à videoaula 1, evidenciando diferença significativa na retenção de informações após a visualização da videoaula construída com base em diretrizes que não sobrecarregam a MT.

As próximas fases da pesquisa trabalharão para identificar o comportamento das funções cognitivas - estresse, engajamento, interesse, entusiasmo, foco e relaxamento - durante a visualização das videoaulas, bem como investigar o nível de retenção de informações na Memória de Longo Prazo, considerando os mesmos cenários de sobrecarga e não sobrecarga cognitiva, o que poderá fornecer um degrau a mais na busca por novos caminhos de análise para a compreensão dos processos cognitivos.

\section{REFERÊNCIAS}

AYRES, P.; SWELLER, J. The split attention principle. In: MAYER, R. (ed.). Cambridge Handbook of Multimedia Learning. New York: Cambridge University Press, 2005.

BADDELEY, A. D. Your memory, a user's guide. New York: Macmillan, 1982. 
BRAGA, A. N. et al. A teoria cognitiva da aprendizagem multimídia no desenvolvimento de atividades de alfabetização matemática. Scientia Plena, v. 15, n. 7, 2019.

CHANDLER, P.; SWELLER, J. Cognitive Load Theory and the Format of Instruction, Cognition and Instruction, v. 8, n. 4, p. 293-332, 1991.

CLARK, R.; NGUYEN, F.; SWELLER, J. Efficiency in Learning: evidence-based guidelines to manage cognitive load. San Francisco: John Wiley \& Sons, 2006.

COOPER, G.; SWELLER, J. The effects of schema acquisition and rule automation on mathematical problem-solving transfer. Journal of Educational Psychology, v. 79, n. 4, p. 347-362, 1987.

COSENZA, R. M.; GUERRA, L. B. Neurociência e Educação: como o cérebro aprende. Porto Alegre: Artmed, 2011.

COWAN, N. The magical number four: how is working memory capacity limited, and why. Current Directions in Psychological Science, v. 19, n. 1, p. 51-57, 2009.

ESTUDOS epidemiológicos - introdução - epidemiologia. [S. d.: s. n.], 2021a. Publicado pelo canal educa.science. Disponível em: https://www.youtube.com/watch?v=MKAJd1c2IJ8. Acesso em: 26 nov. 2021.

ESTUDOS epidemiológicos - resumo - epidemiologia. [S. d.: s. n.], 2021b. Publicado pelo canal educa.science. Disponível em: https://www.youtube.com/watch?v=v3XhyvpnQfs. Acesso em: 26 nov. 2021.

HARP, S.F.; MAYER, R. E. The role of interest in learning from scientific text and illustrations: on the distinction between emotional interest and cognitive interest. Journal of Educational Psychology, v. 89, n. 1, p. 92-102, 1997.

INTRODUÇÃO aos Estudos Epidemiológicos - Resumo - Epidemiologia. [S. d.: s. n.], 2014. Publicado pelo canal Resumed. Disponível em: https://www.youtube.com/watch?v=Jl18GmGOyil. Acesso em: 26 nov. 2021.

JEUNG, H.; CHANDLER, P; SWELLER, J. The role of visual indicators in dual sensory mode instruction. Educational Psychology, v. 17, n. 3, p. 329-343, 1997.

KANDEL, E. R. et al. Princípios de Neurociências. 5. ed. Porto Alegre: AMGH, 2014.

LARKIN, J. S.; SIMON, H. A. Why a diagram is (sometimes) worth a Thousand words. Cognitive Science, v. 11, p. 65-69, 1987.

LAZAR, J.; FENG, J. H.; HOCHHEISER, H. Research methods in human computer Interaction. 2. ed. New Jersey: Wiley, 2017.

LEAHY, W.; CHANDLER; SWELLER, J. When auditory presentations should and should not be a component of multimidia instruction. Applied Cognitive Psychology, v. 17, n. 4, p. 401-418, 2003.

LEVIN, J. Estatística Aplicada a Ciências Humanas. 2. ed. São Paulo: Harbra, 1987.

MAYER, R. E. Cognitive Theory of Multimedia Learning. In: MAYER, R. (ed.). The Cambridge Handbook of Multimedia Learning. New York: Cambridge University, 2005.

MAYER, R. E. Multimedia learning. 2. ed. Santa Barbara: University of California, 2009. 
MILLER, G. The magical number seven, plus or minus two: some limits on our capacity for processing information. Psychological Review, v. 101, n. 2, p. 343-352, 1956.

MORENO, R. Decreasing cognitive load in novice students: effects of explanatory versus corrective feedback in discovery-based multimedia. Instructional Science, v. 32, p. 99-113, 2004.

MOUSAVI, S. Y.; LOW, R.; SWELLER, J. Reducing cognitive load by mixing auditory and visual presentation modes. Journal of Educational Psychology, v. 87, n. 2, p. 319-334, 1995.

PAXIÚBA, C. M. C.; LIMA, C. P. CADAP: Uma Ferramenta de apoio para um Modelo de Avaliação de Aprendizagem Baseado no Desenvolvimento de Conhecimento, Habilidades e Competências e na Reação Emocional dos Alunos. RENOTE: Novas Tecnologias na Educação, Porto Alegre, v. 17, n. 1, jul. 2019.

PETERSON, L.; PETERSON, M. J. Short-term retention of individual verbal items. Journal of Experimental Psychology, v. 58, n. 3, p. 193-198, 1959.

SCHNOTZ, W.; LOWE, R. External and internal representations in multimedia learning. Learning and Instruction, v. 13, n. 2, p. 117-23, 2003.

SHAH, P.; MIYAKE, A. Models of working memory: Mechanisms of active maintenance and executive control. Cambridge: Cambridge University Press, 2003.

SOLSO, R. L. Cognitive Psychology. 4. ed. Boston: Allyn and Bacon, 1995.

SOUZA, N. P. C. Teoria da Carga Cognitiva: origem, desenvolvimento e diretrizes aplicáveis ao processo ensino-aprendizagem. 2010. 175 f. Dissertação (Mestrado em Ciências e Matemáticas)- Universidade Federal do Pará, Belém/PA, 2010.

SWELLER, J. Cognitive load during problem solving: effects on learning. Cognitive Science, p. 257-285, 1988.

TARMIZINI, R.; SWELLER, J. Guidance during mathematical problem solving. Journal of Educational Psychology, v. 80, p. 424-436, 1988.

TINDALL-FORD, S.; CHANDLER, P.; SWELLER, J. When two sensory modes are better than one. Journal of Experimental Psychology: Applied, v. 3, n. 4, p. 257-287, 1997.

VAN MERRIENBOER, J. J. G.; KESTER, L.; PAAS. F. Teaching complex rather than simple tasks: Balancing intrinsic and germane load to enhance transfer of learning. Applied Cognitive Psychology, v. 20, n. 3, p. 343-352, 2006.

WARD, M.; SWELLER, J. Structuring effective worked examples. Cognition and Instruction, v. 7, n. 1, p. 139, 1990.

ZAR, T.; GALERA, C. A. A dinâmica temporal da atenção na memória de trabalho visual. Psico, Porto Alegre, v. 49, n. 4, p. 358-364, 2018. 